\title{
Trial Application of Pulse-Field Magnetization to Magnetically Levitated Conveyor System
}

\author{
Yoshihito Miyatake, Mochimitsu Komori, Ken-ichi Asami, and Nobuo Sakai \\ Department of Applied Science for Integrated System Engineering, Kyushu Institute of Technology, 1-1 Sensui, Tobata, Kitakyushu, \\ Fukuoka 804-8550, Japan \\ Correspondence should be addressed to Mochimitsu Komori, komori_mk@yahoo.co.jp
}

Received 5 May 2012; Accepted 15 July 2012

Academic Editor: Masato Murakami

Copyright (๑) 2012 Yoshihito Miyatake et al. This is an open access article distributed under the Creative Commons Attribution License, which permits unrestricted use, distribution, and reproduction in any medium, provided the original work is properly cited.

Magnetically levitated conveyor system using superconductors is discussed. The system is composed of a levitated conveyor, magnetic rails, a linear induction motor, and some power supplies. In the paper, pulse-field magnetization is applied to the system. Then, the levitation height and the dynamics of the conveyor are controlled. The static and dynamic characteristics of the levitated conveyor are discussed.

\section{Introduction}

There are some kinds of levitation systems using electromagnetic forces, such as a force between electromagnet and magnetic material, a force between electromagnet and permanent magnet, and a force between superconductor and permanent magnet [1-4]. These methods have advantages for their contact-free conditions. However, disadvantages are there for each system. In this paper, we made a magnetically levitated conveyor using electromagnetic forces between superconductors and permanent magnets [5]. Superconducting levitation technologies have advantages that the system does not need complicated systems such as control system. And it is easy for the system to levitate stably. However, there is a problem that the levitation height and the dynamics change as time passes. This is because magnetic fluxes move to another location due to flux creep effect.

In order to solve the problem, pulse-field magnetization is applied to the system to improve the levitation height and the dynamics of the levitated conveyor. A magnetic analysis for the system was performed to make a better structure of the system. The analysis of the system leads to an improved system and the system is studied experimentally in this paper.

\section{System}

2.1. Total System. Figure 1 shows a total illustration of the magnetically levitated conveyor system. The system consists of a levitated conveyor, a pair of magnetic rails, two electromagnets for pulse-field magnetization and driving the levitated conveyor, and a power supply for pulse-field magnetization. The $x y z$-coordinates are indicated as shown in the figure.

2.2. Magnetic Rail. Figure 2 shows a cross-section of the magnetic rails with a linear induction motor. The magnets of rails are made of NdFeB materials and the specifications of the magnets are shown in Table 1. As shown in Figure 2, a rail is composed of two magnets facing each other. Then, the magnetic poles face each other. Yoke material is inserted in between the magnets to increase a magnetic flux density over the central part of the magnetic rail. Furthermore, the yoke material has some effects to unify the magnetic flux density along the magnetic rail and to relax the discontinuity of the magnetic flux density at the magnet joints.

2.3. Driving Structure. An illustration of the linear induction motor used for the system is shown in Figure 3. 


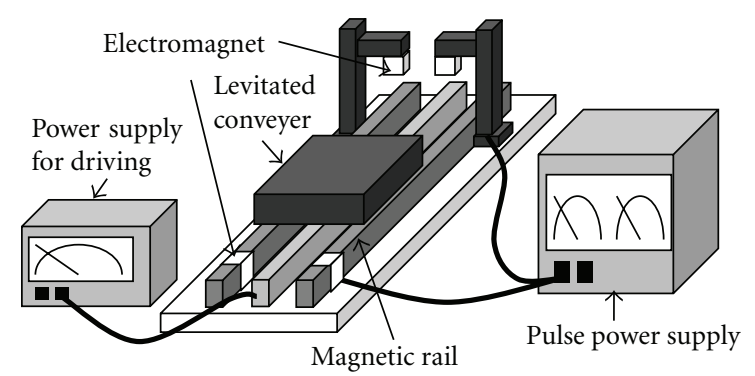

Figure 1: System.

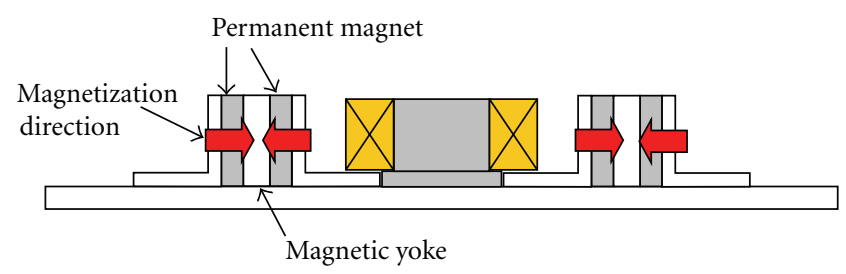

FIgURe 2: Magnetic rail.

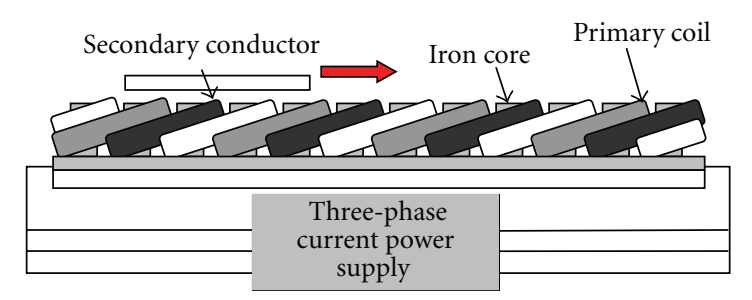

Figure 3: Linear induction motor.

TABLE 1: Specifications of the permanent magnet.

\begin{tabular}{lc}
\hline Materials & NdFeB \\
\hline Dimensions & $35 \times 20 \times 5(\mathrm{~mm})$ \\
Surface magnetic flux density & $0.31(\mathrm{~T})$ \\
Coercive force & $868(\mathrm{KA} / \mathrm{m})$ \\
\hline
\end{tabular}

TABLe 2: Specifications of linear induction motor.

\begin{tabular}{lcc}
\hline Item & Numerical value & Unit \\
\hline Iron core (one) & $10 \times 20 \times 16$ & $\mathrm{~mm}$ \\
Wire diameter & 0.4 & $\mathrm{~mm}$ \\
Turn number & 80 & Turn \\
Wire resistance & 2.5 & $\Omega$ \\
Inductance & 8.36 & $\mathrm{mH}$ \\
\hline
\end{tabular}

The induction motor is composed of a primary coils and an induction plate with three phase to drive the levitated conveyor. The color of primary coil shows the same phase. The windings of three phase is surrounded by every two primary iron core. The specifications of the linear induction motor are shown in Table 2.

2.4. Electromagnet. The illustration of magnetically levitated conveyor with electromagnets for pulse-field magnetization

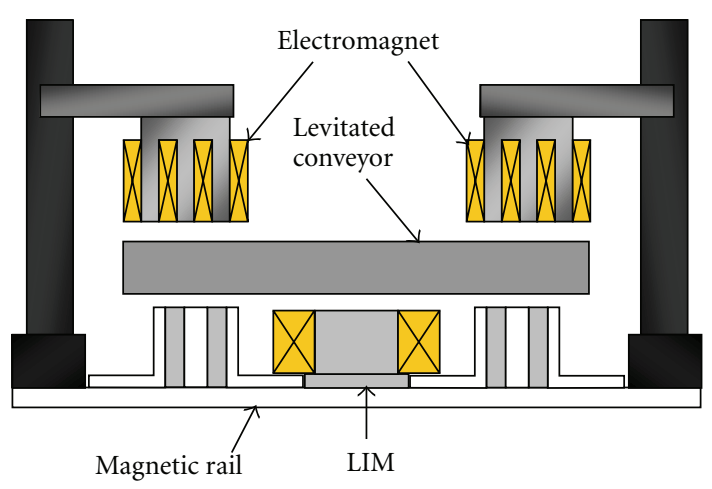

Figure 4: Magnetization method.

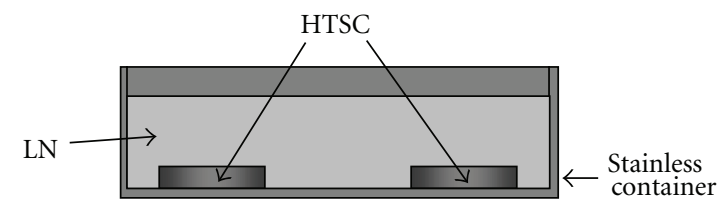

FIgURE 5: Cross-section of the levitated conveyer.

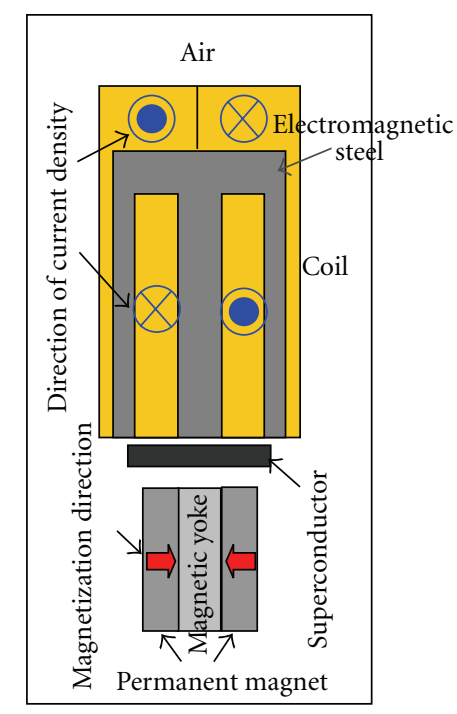

FIgURE 6: Model of magnetization of opposite direction. 


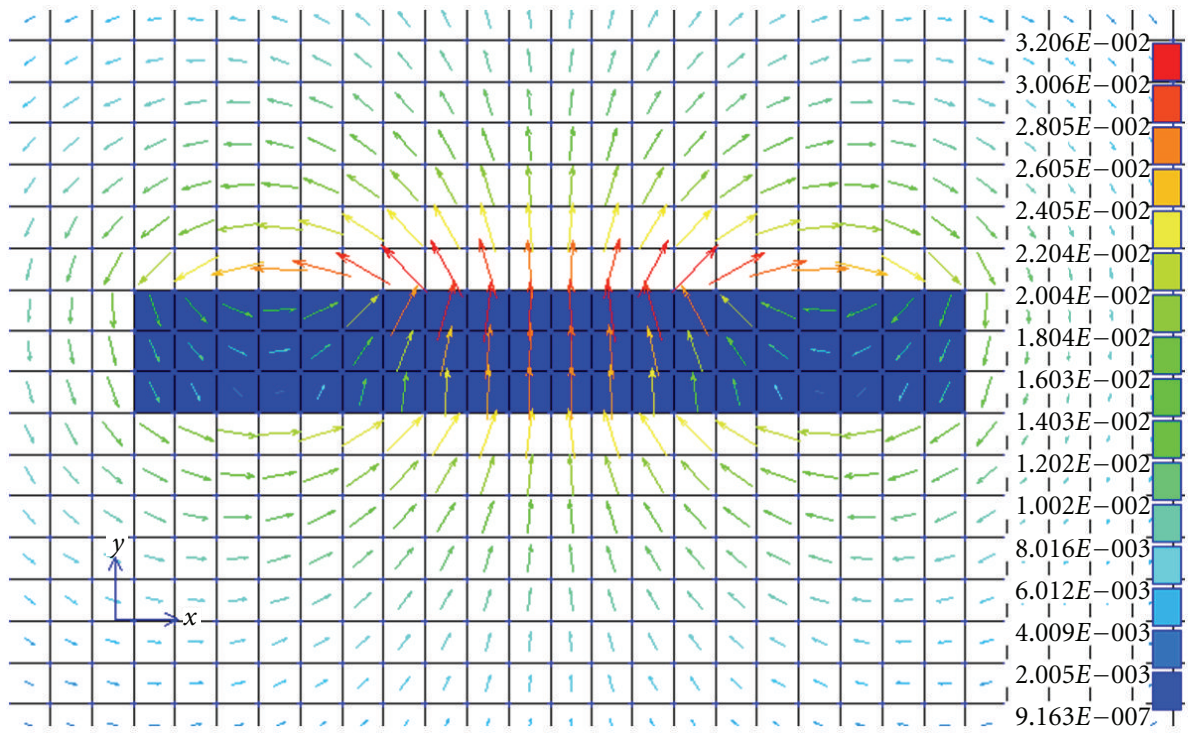

Figure 7: Analytical result of magnetic flux density vector after the magnetization in the same direction.

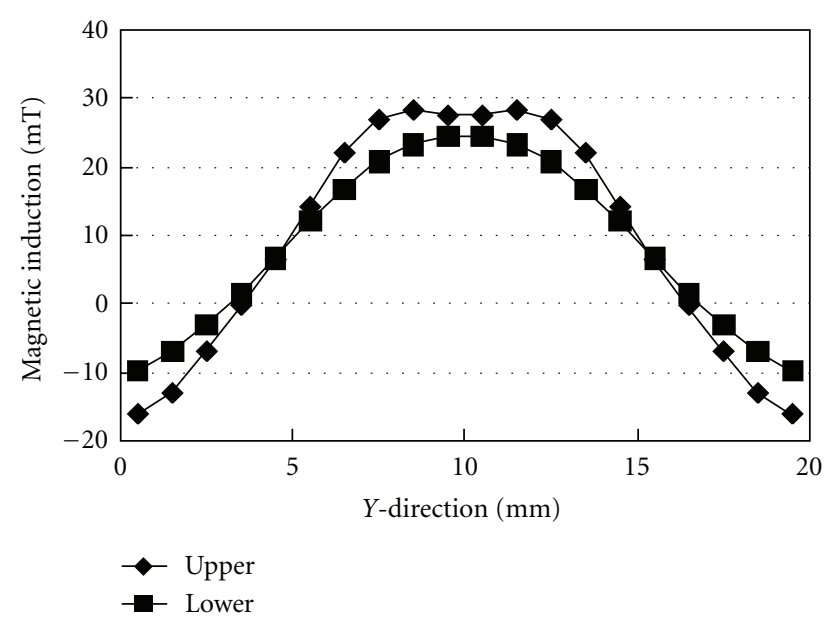

Figure 8: Magnetic flux density at the surface of superconductor magnetized in the same direction.

is shown in Figure 4. By installing bigger electromagnets over the levitated conveyor, pulse-field magnetization is easily applied to superconductors in the levitated conveyor and larger pulse-field magnetization is easily obtained. Since the magnetic flux density distributions in superconductors are uniform along the magnetic rails, it is necessary for the electromagnets to produce magnetic flux densities similar to those from the magnetic rails. The specifications of electromagnets are shown in Table 3.

2.5. Levitated Conveyor. The levitated conveyor is shown in Figure 5. The conveyor is a rectangular container made of stainless steel for saving liquid nitrogen. Four superconductors are installed at the bottom of the container for levitation. The specifications of the superconductor are
TABLE 3: Specifications of electromagnet.

\begin{tabular}{lcc}
\hline Item & Numerical value & Unit \\
\hline Coil (one) & $16 \times 12 \times 24$ & $\mathrm{~mm}$ \\
Core & $0.5 \times 40 \times 24$ & $\mathrm{~mm}$ \\
Wire diameter & 0.8 & $\mathrm{~mm}$ \\
Turn number & 225 & Turn \\
Wire resistance & 1 & $\Omega$ \\
Inductance & 3 & $\mathrm{mH}$ \\
\hline
\end{tabular}

TABLE 4: Specifications of the superconductor.

\begin{tabular}{lcc}
\hline Item & Numerical value & Unit \\
\hline Composition & $\mathrm{SmBaCuO}$ & - \\
Dimensions & $\phi 20 \times 3.2 t$ & $\mathrm{~mm}$ \\
Critical current density $J_{c}$ & $2.7 \times 10^{8}($ at $1 \mathrm{~T})$ & $\mathrm{A} / \mathrm{m}^{2}$ \\
Critical temperature $T_{c}$ & 92 & $\mathrm{~K}$ \\
\hline
\end{tabular}

shown in Table 4. In the experiment, the superconductors are field cooled by using liquid nitrogen.

\section{Analysis}

Figure 6 shows a simulation model representing a crosssection of the conveyor system with electromagnets for pulse-field magnetization electromagnets. It is assumed that the distance between superconductors in the levitated conveyor and the magnetic rails is $5.0 \mathrm{~mm}$. The distance between superconductors and electromagnets is $1.0 \mathrm{~mm}$ because there is a buffer spacer between superconductors and electromagnets. In the simulation, relative permeabilities of iron core and electromagnet steel are 5,000 and 18,000, respectively. 


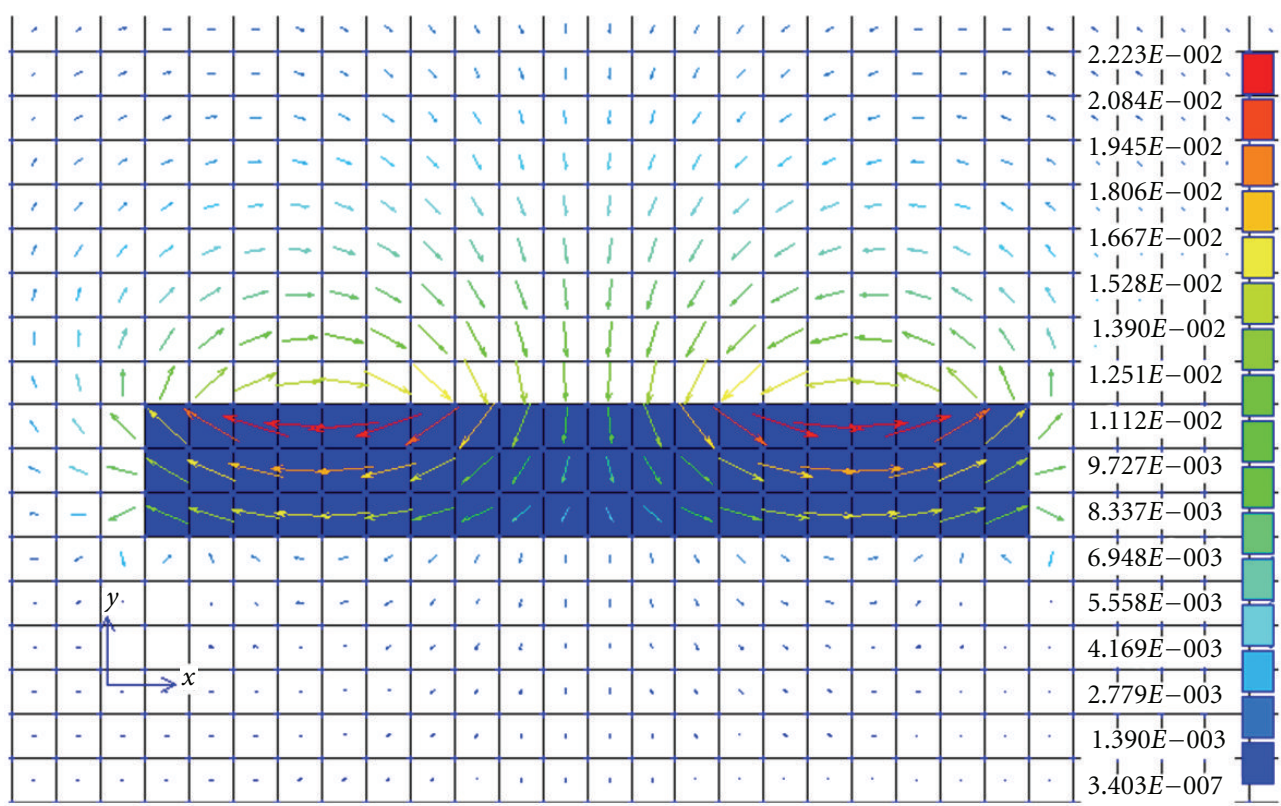

FIGURE 9: Analytical result of flux density after the magnetization in the opposite direction.

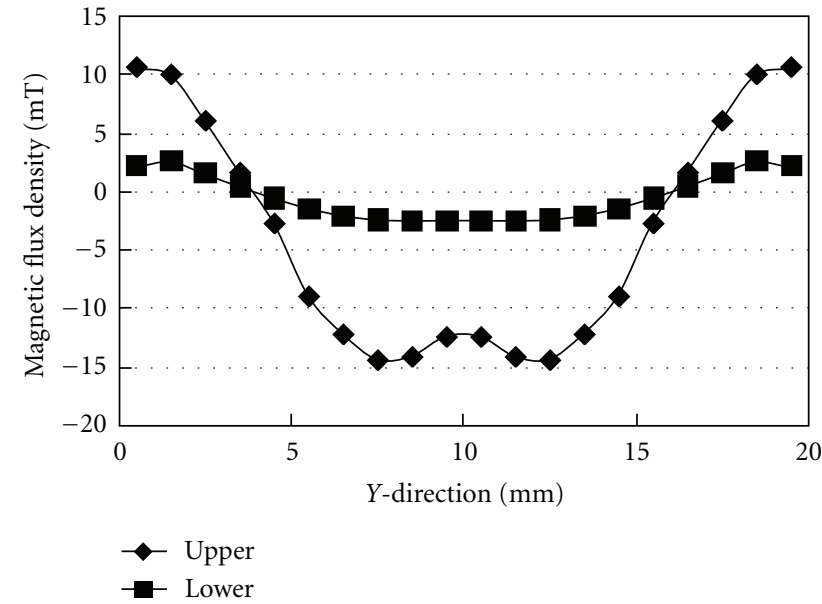

Figure 10: Magnetic flux density of upper part direction after the magnetization in the opposite direction.

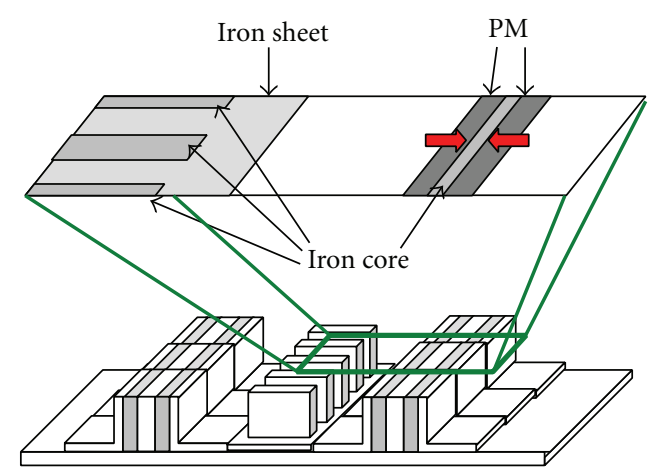

FIGURE 11: Measurement experiment of magnetic field distribution of magnetic rail.

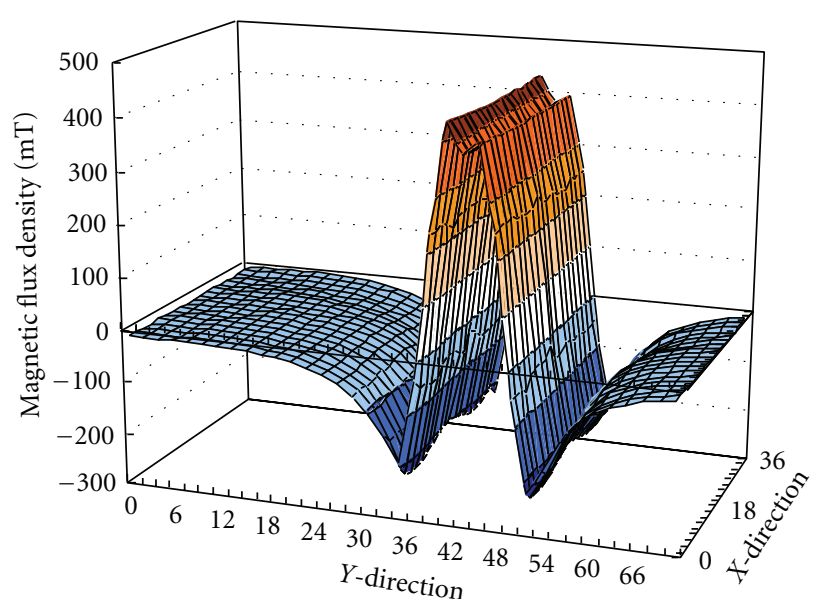

FIGURE 12: Magnetic field distribution of magnetic rail.

In the case of pulse-field magnetization in the same direction as that of the magnetic rails, the magnetic flux densities in the superconductors are simulated. The magnetic flux density distribution is shown in Figure 7. The length and arrow of vectors mean the magnitude and direction of magnetic flux density, respectively. As shown in the figure, the superconductor is magnetized in the upper direction. Figure 8 shows the magnetic flux densities at the upper and lower superconductor surfaces magnetized in the same direction. It is found that the magnetic flux densities become large in the center of superconductor and that the difference between upper and lower superconductor surfaces is not so large. The simulation results show that it is not so difficult to magnetize the superconductor which is already magnetized by using magnetic rails. 


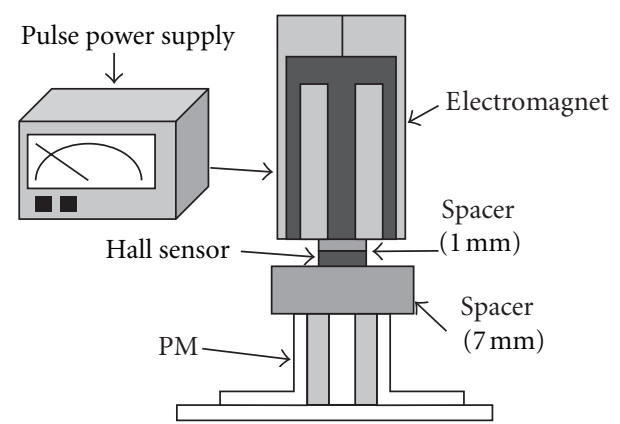

FIGURE 13: Measurement experiment of magnetic field.

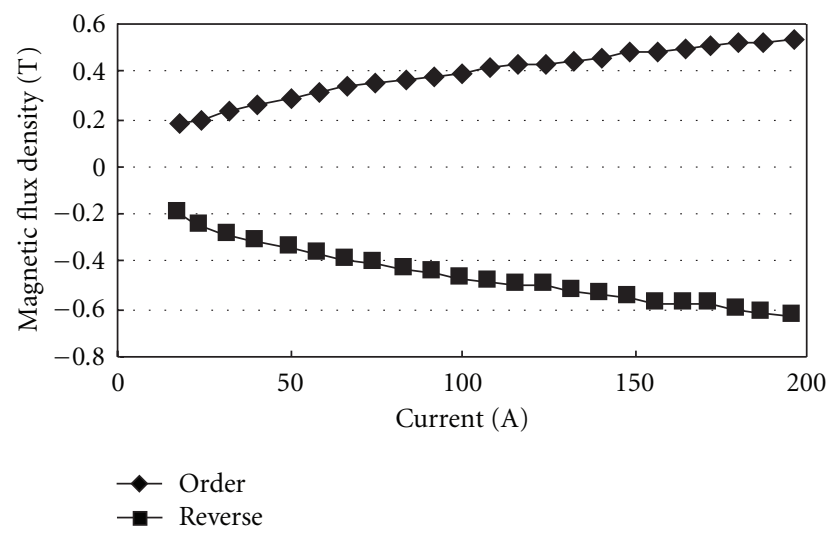

FIGURE 14: Measurement result of magnetic field.

In the case of pulse-field magnetization in the opposite direction to that of the magnetic rails, the magnetic flux densities in the superconductors are simulated. Figure 9 shows the analytical result of magnetic flux density distribution. As shown in the figure, the superconductor is magnetized in the opposite direction to the magnetic rails. Figure 10 shows the magnetic flux densities at the upper and lower superconductor surfaces magnetized in the opposite direction. It is found that the magnetic flux densities at the upper superconductor surface become large in the center of the superconductor and the difference between upper and lower superconductor surfaces is a little large as compared with Figure 8. These results show that the magnetization by using the electromagnet over superconductor is successful.

\section{Experiment}

4.1. Magnetic Flux Density Distribution of Magnetic Rail. The magnetic flux densities were measured at a distance $1.0 \mathrm{~mm}$ from the magnetic rail surface. Figure 11 shows the measured area of the magnetic rail. The measured area consists of a magnetic rail and a half of the LIM. Figure 12 shows the magnetic flux density distribution at a distance $1.0 \mathrm{~mm}$ from the rail. The result shows that there is a peak of magnetic flux density around $y=40 \mathrm{~mm}$ and that the distribution is almost the same in the $x$-direction. Therefore, it is possible for the conveyor to levitate stably and move smoothly in the $x$-direction. The influence of the linear induction motor is regarded as small.

4.2. Magnetic Field of Electromagnet. Figure 13 shows an experimental setup for measuring magnetic flux density. The setup consists of a magnetic rail, an electromagnet, two spacers, and a pulse power supply. The spacer of $7.0 \mathrm{~mm}$ corresponds to the superconductor $(3.0 \mathrm{~mm})$, a gap $(3.5 \mathrm{~mm})$ and the container thickness $(0.5 \mathrm{~mm})$. The measurement was performed at a distance $1.0 \mathrm{~mm}$ from the magnetic rail. In other words, the Hall sensor is arranged on the upper superconductor surface. Figure 14 shows the result of magnetic flux density distribution. The initial magnetic flux density due to the magnetic rail is about $180 \mathrm{mT}$. The maximum magnetic flux densities in the same and opposite directions are both about $0.6 \mathrm{~T}$, which is relatively larger than that of previous electromagnets. The length and resistance of copper wire become shorter and smaller than the previous electromagnets, respectively. Anyhow, the magnetic flux density increases both in the case of the same and opposite directions.

4.3. Trapped Magnetic Flux Density. Figure 15 shows the magnetic flux density distributions at a distance $1.0 \mathrm{~mm}$ from (a) the upper superconductor surface and from (b) the lower superconductor surface, corresponding to the condition where the distance between lower superconductor surface and magnetic rails are $5.0 \mathrm{~mm}$. As shown in Figure 15, there is a magnetic flux peak for each distribution. The maximum magnetic flux density in Figure 15(b) is larger than that of the upper superconductor surface in Figure 15(a). This is because the magnetic rails produce the magnetic flux density at the lower superconductor surface more than that at the upper superconductor surface.

Figure 16 shows the distribution of magnetic flux density of the superconductor after pulse-field magnetization in the same direction as that of magnetic rails, showing the distribution at (a) the upper superconductor surface and (b) the lower superconductor surface. The pulse field was applied five times to the superconductors at a supply voltage $250 \mathrm{~V}$. Each distribution has two peaks of magnetic flux density. The maximum densities are around $150 \mathrm{mT}$. By using pulse-field magnetization, the magnetic flux densities in Figures 16(a) and 16(b) are larger than those in Figures 15(a) and 15(b), respectively. This means that the pulse-field magnetization in the same direction as the magnetic rails increases the initial magnetization. Figure 17 shows the distribution of magnetic flux density of the superconductor after pulse-field magnetization in the opposite direction to that of magnetic rails, showing the distribution at (a) the upper superconductor surface and (b) the lower superconductor surface. The magnetic flux density at the upper superconductor surface in Figure 17(a) becomes small compared with that in Figure 15(a). The magnetic flux density at the lower superconductor surface in Figure 17(b) becomes a little smaller than that in Figure 15(b). By using pulse-field magnetization, the magnetic flux densities in Figures 17(a) and 17(b) become smaller than those 

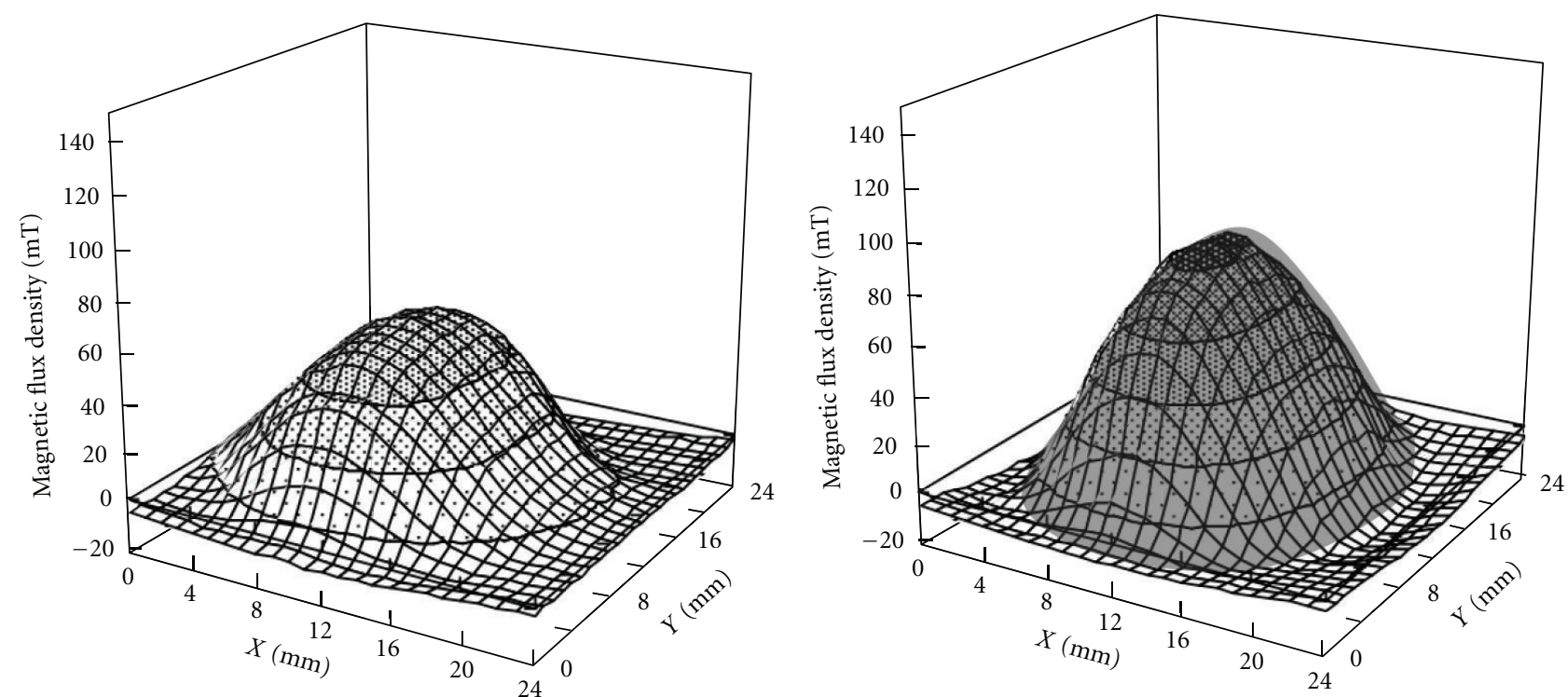

\begin{tabular}{|c|c|}
\hline $120-140$ & 堅 $40-60$ \\
\hline $100-120$ & $\because 20-40$ \\
\hline $80-100$ & ․ $0-20$ \\
\hline $60-80$ & $-20-0$ \\
\hline
\end{tabular}

\begin{tabular}{|c|c|}
\hline $120-140$ & 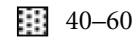 \\
\hline $100-120$ & $\because 20-40$ \\
\hline $80-100$ & ․ $0-20$ \\
\hline $60-80$ & $-20-0$ \\
\hline
\end{tabular}

(a)

(b)

FIGURE 15: Distribution of magnetic flux density before magnetizing.
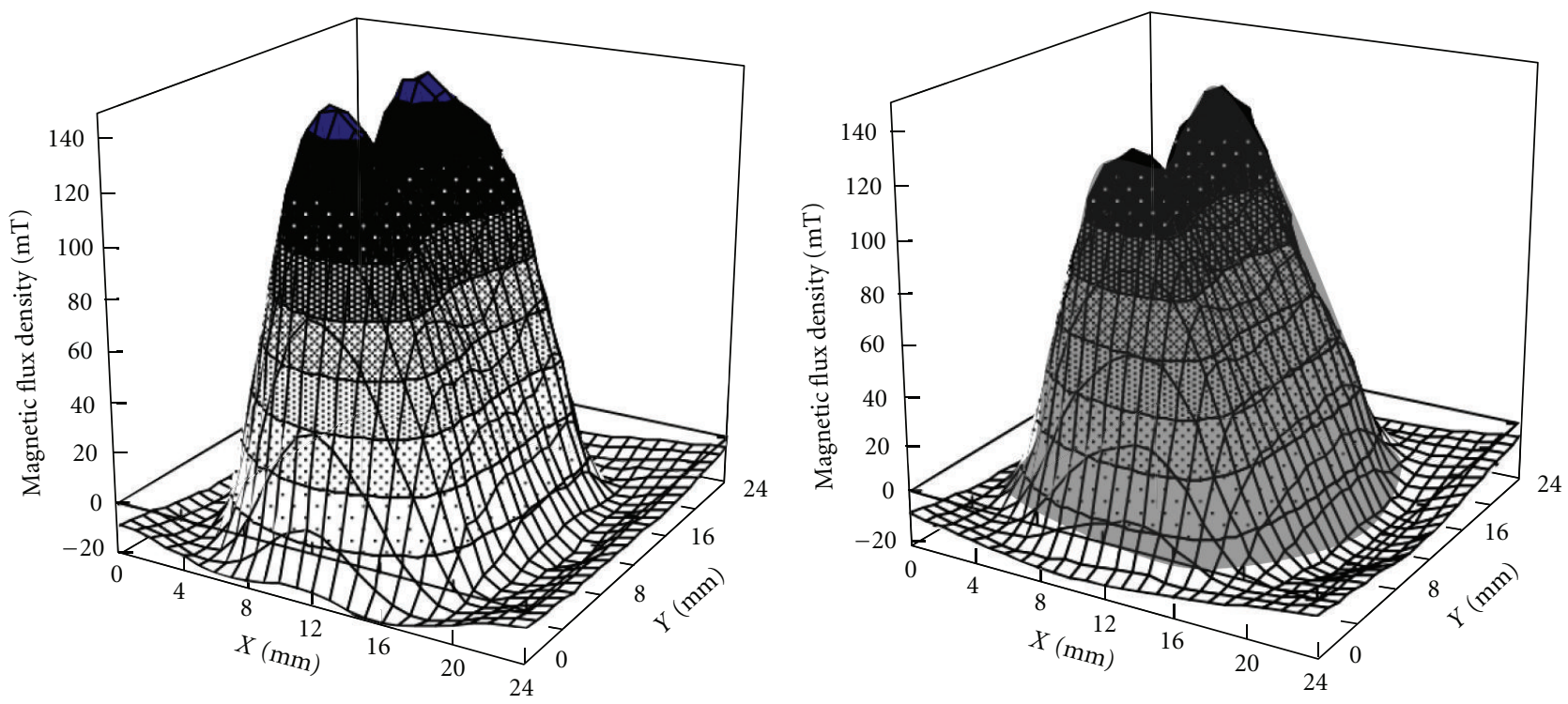

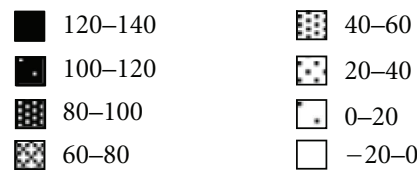

(a)

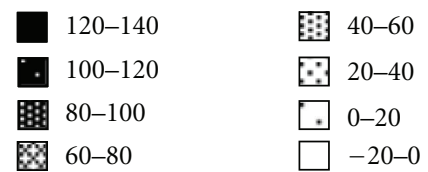

(b)

FIGURE 16: Distribution of magnetic flux density after the magnetization in the same direction. 

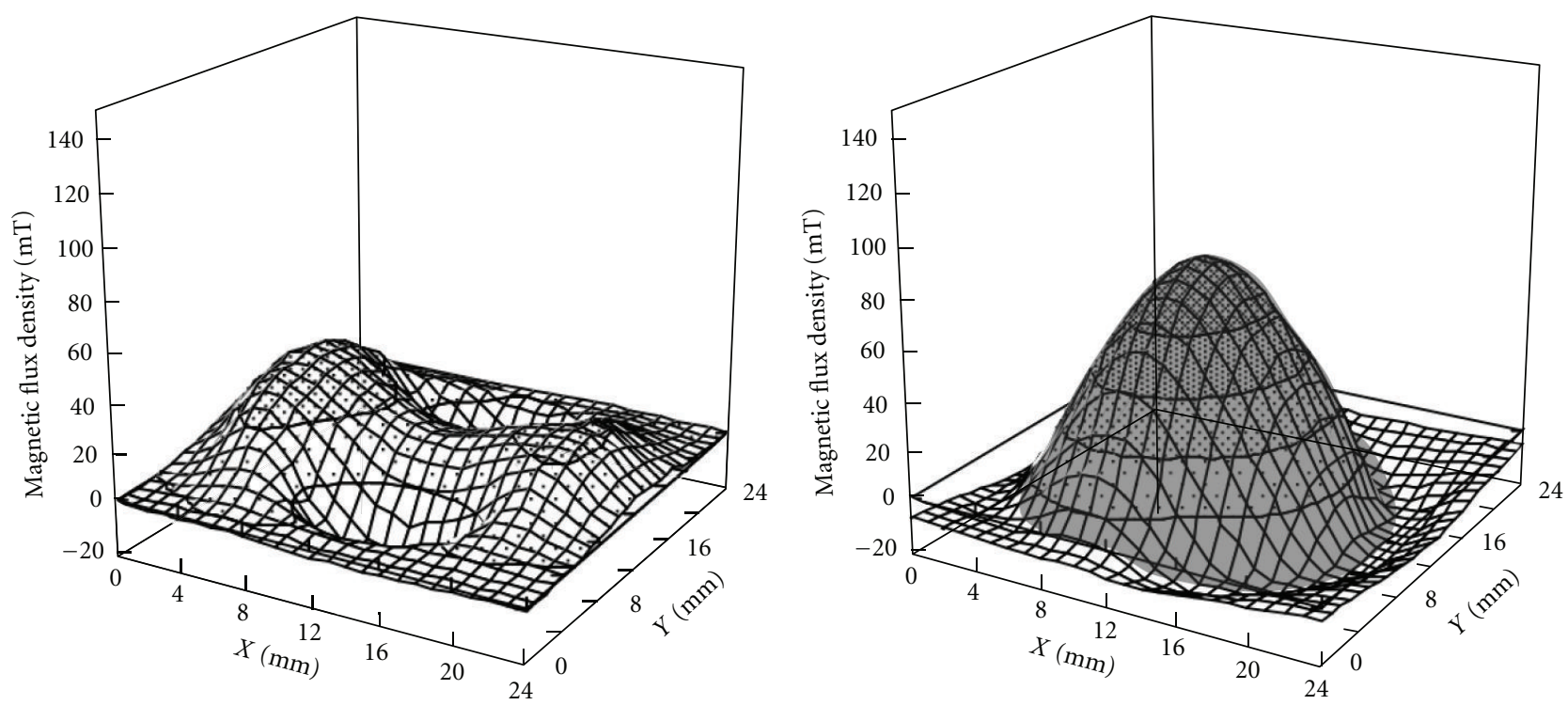

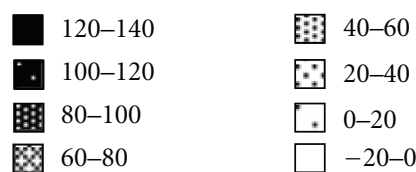

(a)

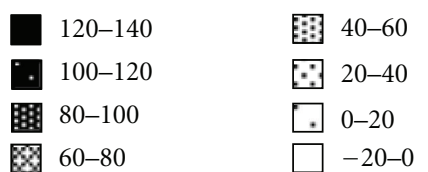

(b)

FIGURE 17: Distribution of magnetic flux density after the magnetization in the opposite direction.

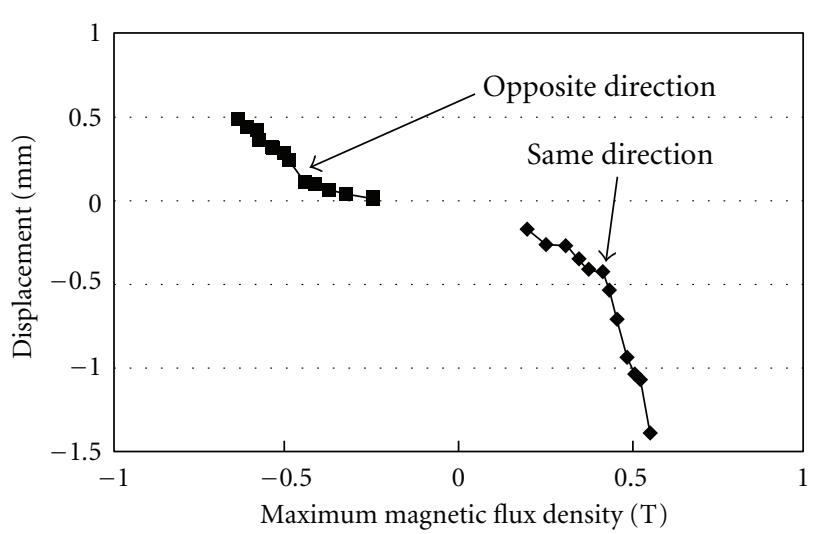

FIgURE 18: Displacement of levitating height.

in Figures 15(a) and 15(b), respectively. This means that the pulse-field magnetization in the opposite direction to the magnetic rails decreases the initial magnetization. These results show that the effect of field magnetization in the same direction as magnetic rails is larger than the effect of field magnetization in the opposite direction.

4.4. Levitation Height. The levitation height of levitated conveyor was measured after pulse-field magnetizations in the same and opposite directions. Figure 18 shows the displacement of the levitated conveyor after pulse-field magnetizations. The measurement was performed by using a laser displacement sensor. The displacement zero corresponds to the initial position of the levitated conveyor. As shown in Figure 18, the displacement changes after the pulsefield magnetization is applied. The displacement which is changed in the same direction is larger than that in the opposite direction. This is because the change of magnetic flux density in Figure 16(b) is larger than that in Figure 17(b).

4.5. Impulse Response. Impulse responses for the levitated conveyor were performed after pulse-field magnetizations in the same and opposite directions. Just after impulses are applied to the levitated conveyor, the displacement of the conveyor is measured in the $z$-direction by using a laser displacement sensor. Then, spring constant and damping coefficient are evaluated before and after the field magnetizations. Table 5 shows the spring constant and the damping coefficient. The spring constant $4,964 \mathrm{~N} / \mathrm{m}$ and damping coefficient $4.45 \mathrm{Ns} / \mathrm{m}$ after field magnetization in the same direction are larger than the initial spring constant $2,574 \mathrm{~N} / \mathrm{m}$ and damping coefficient $2.87 \mathrm{Ns} / \mathrm{m}$, respectively. On the other hand, the spring constant 2,183 N/m and the damping coefficient $2.67 \mathrm{Ns} / \mathrm{m}$ in the opposite direction are almost the same as the initial spring constant and initial damping coefficient, respectively. 
TABLE 5: Measurment results of impulse response.

\begin{tabular}{lcc}
\hline & $\begin{array}{c}\text { Spring constant } \\
(\mathrm{N} / \mathrm{m})\end{array}$ & $\begin{array}{c}\text { Damping } \\
\text { coefficient }(\mathrm{Ns} / \mathrm{m})\end{array}$ \\
\hline Before magnetization & 2574 & 2.87 \\
Same direction $(250 \mathrm{~V})$ & 4964 & 4.45 \\
Opposite direction $(250 \mathrm{~V})$ & 2183 & 2.67 \\
\hline
\end{tabular}

\section{Summary}

Superconducting levitation characteristics depend on their initial condition, such as the initial levitation height. Thus, we proposed a new superconducting levitation technique, magnetically levitated linear conveyor system using hybrid magnetization. The pulse-field magnetization in the same direction as the magnetic rails is more effective than in the opposite direction.

The pulse-field magnetization from the upper superconductor surface of the levitated conveyor is useful for the system. From the experimental results, it is found that the pulse-field magnetization at the upper superconductor surface changes the initial magnetic flux density more than magnetic flux density at the lower surface.

The pulse-field magnetization technique leads to controlling the conveyor conditions depending on the height, the speed, and so on. This is applicable to a full-scale working model. Dynamic characteristics of the levitated conveyor will be discussed in the next paper.

\section{References}

[1] J. R. Hull, T. M. Mulcahy, K. L. Uherka, and R. G. Abboud, "Low rotational drag in high-temperature superconducting bearings," IEEE Transactions on Applied Superconductivity, vol. 5, no. 2, pp. 626-629, 1995.

[2] M. Komori, H. Kobayashi, and M. Kumamoto, "Hybrid high $\mathrm{T}_{\mathrm{c}}$ superconducting magnetic bearing (SMB) system with no bias currents," in Proceedings of the 6th International Symposium on Magnetic Bearings, pp. 214-223, MIT, Cambridge, Mass, USA, August 1998.

[3] T. Ichihara, K. Matsunaga, M. Kita et al., "Application of superconducting magnetic bearings to a $10 \mathrm{kWh}$-class flywheel energy storage system," IEEE Transactions on Applied Superconductivity, vol. 15, no. 2, pp. 2245-2248, 2005.

[4] H. Kojima, O. Itagaki, T. Okabe, and T. Kobayashi, "Study on non-contact hold and transfer control of spherical magnetic body by magnetic robust hand with prototype gap sensor system," Journal of the Robotics Society of Japan, vol. 14, pp. 868874, 1996.

[5] M. Komori and G. I. Kamogawa, "Basic study of a magnetically levitated conveyer using superconducting magnetic levitation," IEEE Transactions on Applied Superconductivity, vol. 15, no. 2, pp. 2238-2241, 2005. 

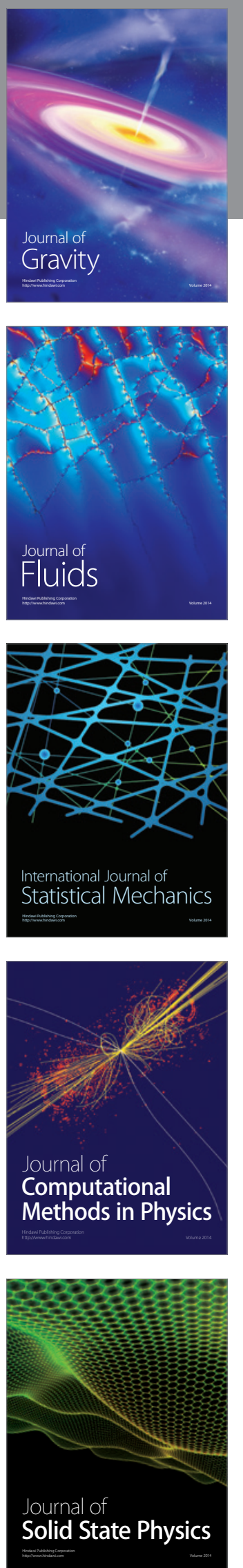

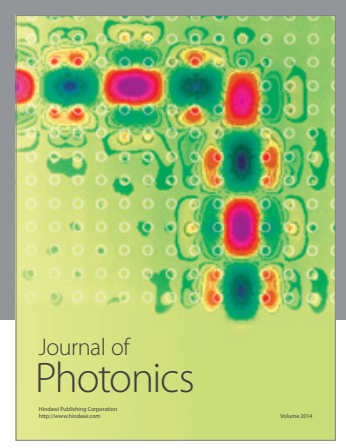

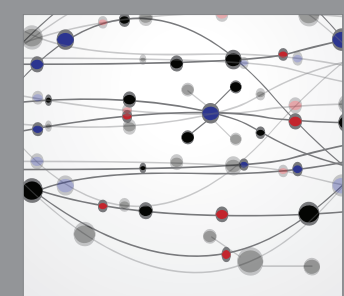

The Scientific World Journal
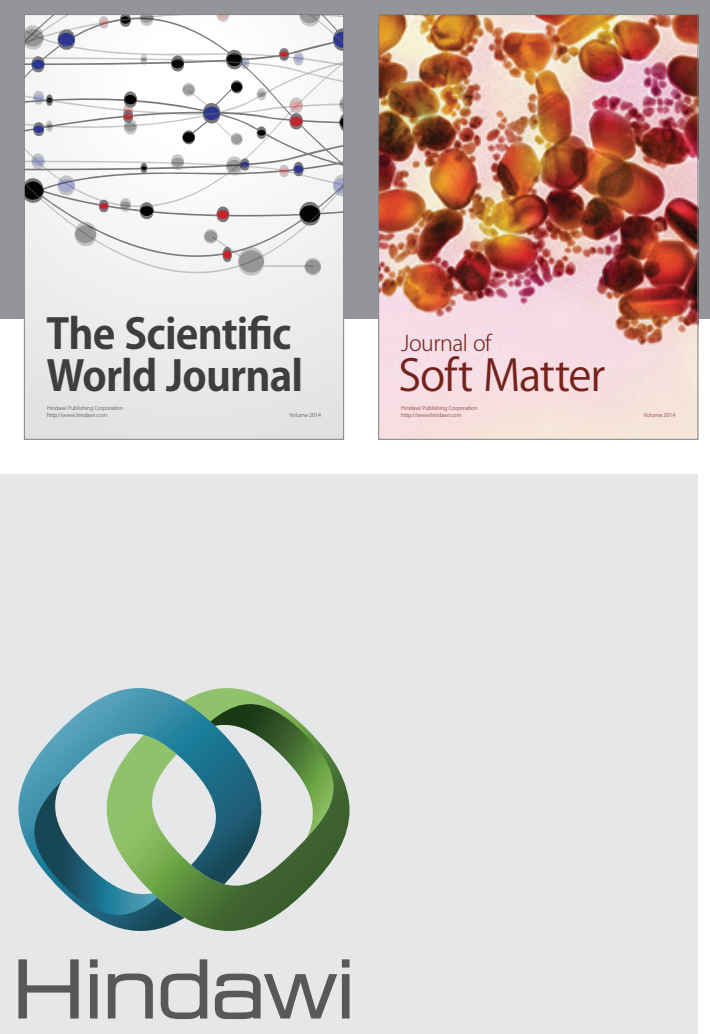

Submit your manuscripts at

http://www.hindawi.com
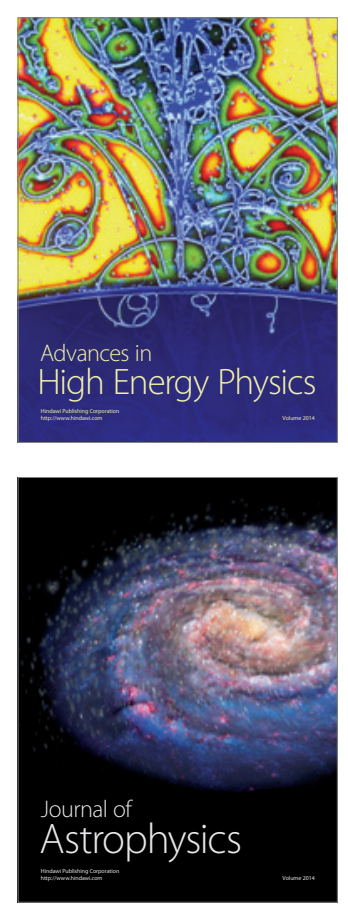
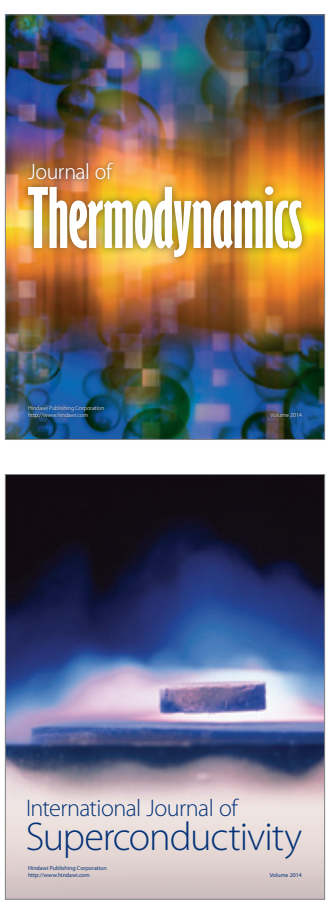
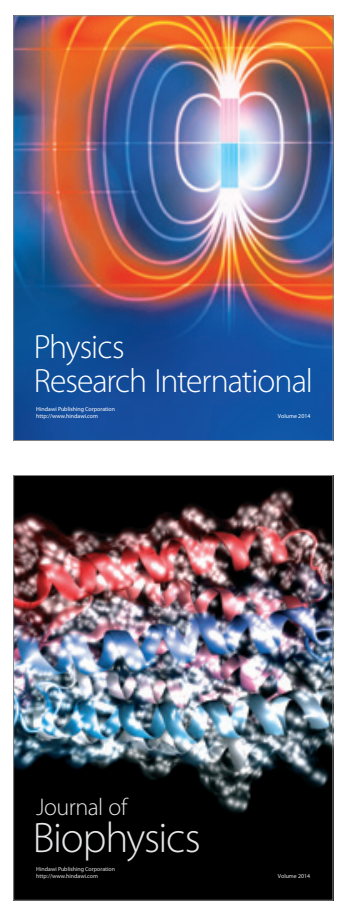
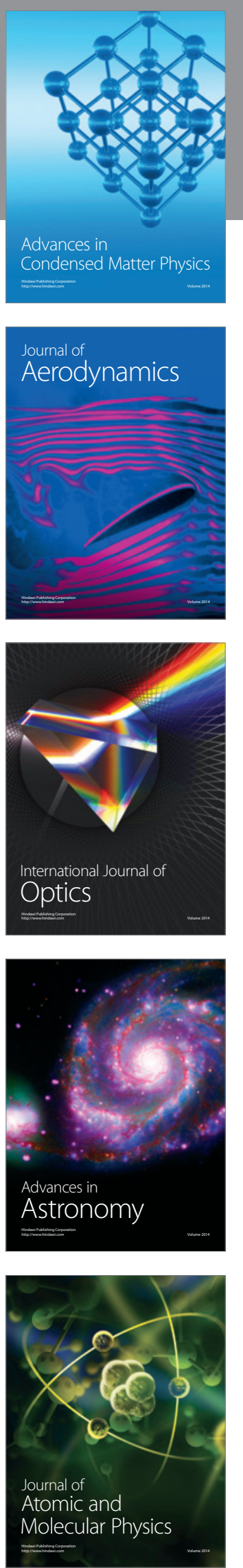